\title{
Predictive Value of Body Posture and Pupil Dilation in Assessing Consumer Preference and Choice
}

Ramsøy, Thomas Zoëga ; Jacobsen, Catrine; Friis-Olivarius, Morten; Bagdziunaite, Dalia; Skov, Martin

\author{
Document Version \\ Accepted author manuscript \\ Published in: \\ Journal of Neuroscience, Psychology, and Economics
}

DOI:

10.1037/npe0000073

10.1037/npe0000073.supp

Publication date:

2017

License

Unspecified

Citation for published version (APA):

Ramsøy, T. Z., Jacobsen, C., Friis-Olivarius, M., Bagdziunaite, D., \& Skov, M. (2017). Predictive Value of Body Posture and Pupil Dilation in Assessing Consumer Preference and Choice. Journal of Neuroscience,

Psychology, and Economics, 10(2/3), 95-110. https://doi.org/10.1037/npe0000073,

https://doi.org/10.1037/npe0000073.supp

Link to publication in CBS Research Portal

\section{General rights}

Copyright and moral rights for the publications made accessible in the public portal are retained by the authors and/or other copyright owners and it is a condition of accessing publications that users recognise and abide by the legal requirements associated with these rights.

\section{Take down policy}

If you believe that this document breaches copyright please contact us (research.lib@cbs.dk) providing details, and we will remove access to the work immediately and investigate your claim. 


\title{
Predictive value of body posture and pupil dilation in assessing consumer preference and choice
}

\section{Thomas Zoëga Ramsøy, Catrine Jacobsen, Morten Friis-Olivarius, Dalia Bagdziunaite, and Martin Skov}

\author{
Journal article (Accepted manuscript)
}

CITE: Ramsøy, T. Z., Jacobsen, C., Friis-Olivarius, M., Bagdziunaite, D., \& Skov, M. (2017). Predictive value of body posture and pupil dilation in assessing consumer preference and choice. Journal of Neuroscience, Psychology, and Economics, 10(2-3), 95-110. http://dx.doi.org/10.1037/npe0000073

(CAmerican Psychological Association, 2017. This paper is not the copy of record and may not exactly replicate the authoritative document published in the APA journal. Please do not copy or cite without author's permission. The final article is available, upon publication, at: http://dx.doi.org/10.1037/npe0000073

Uploaded to Research@CBS: December २०18 
Predictive value of body posture and pupil dilation in assessing consumer preference and choice 


\begin{abstract}
In neuroeconomics and neuromarketing, the assessment of arousal has become one of the key measures in our effort to understand the basic mechanisms of value-based choice. While neurophysiological responses such as pupil dilation and galvanic skin response (GSR) have provided a significant explanatory value in our understanding of decision making, other, less known responses such as body posture, may provide additional explanatory value. Here, we report the results from three separate high-resolution eye-tracking studies in which pupil dilation and body posture provide both independent and interacting contributions in predicting preference judgments and choice. Notably, our data suggest that a combination of pupil dilation and posture can be used to assess arousal and valence, respectively, and thus provide a better estimate of emotional responses to stimuli, and their effect on choice. However, the use of this combined measure needs to be used with care, as the dynamic relationship between pupil size and posture is affected by different categories of stimuli. We discuss these findings in light of the academic and commercial call for neuroimaging and physiology measures that can predict and explain the causal mechanisms underlying preference formation and value based choice.
\end{abstract}

Word count: 192 


\section{Introduction}

How can we predict preference and choice? In recent years, the integration of neurobiological models and methods into classical economic domains has been on the rise (Plassmann, Ramsøy, \& Milosavljevic, 2012) leading to the nascent and overlapping fields of neureonomics (Braeutigam, 2005; Camerer, Loewenstein, \& Prelec, 2005; Kenning \& Plassmann, 2005; Rustichini, 2005), decision neuroscience (Mohr, Biele, Krugel, Li, \& Heekeren, 2010; Wunderlich, Rangel, \& O'Doherty, 2010), neuromarketing (Ariely \& Berns, 2010; Fisher, Chin, \& Klitzman, 2010; Lee, Broderick, \& Chamberlain, 2007; Senior \& Lee, 2008), and consumer neuroscience (Hubert \& Kenning, 2008; Plassmann, Kenning, Deppe, Kugel, \& Schwindt, 2008). One motivation for this multidisciplinary effort is the belief that an understanding of the neural mechanisms underlying preference formation, judgments, and decision-making will lead to an improved ability to predict preferences and choice behavior.

The attempt to predict behavior from physiological data can be traced back to earlier studies in psychology and psychophysiology, including the controversial studies by Benjamin Libet (Libet, 1999; Libet, 2002; Libet, Gleason, Wright, \& Pearl, 1983). Libet demonstrated a dissociation between brain responses and the conscious sense of willed action. In particular, Libet demonstrated that brain activation, as studied by electroencephalography (EEG), occurred up to 500 milliseconds before subjects reported having a conscious experience of making a choice. Although Libet's studies have been criticized on methodological grounds (e.g., Churchland, 1981; Klein, 2002), more recent and methodologically sound studies have demonstrated that brain responses can indeed predict choices up to several seconds in advance (Soon, Brass, Heinze, \& Haynes, 2008). 
The finding that choice behavior appears to be initiated by neural processes that are not experienced consciously, well in advance of the on-set of behavior, has spurn a widespread interest in examining these unconscious processes more closely. An example of this is the link between arousal and risky choices seen in studies using the Iowa Gambling Task. Here, increased arousal, as assessed by galvanic skin response (GSR), has been shown to occur prior to all choices. With repeated exposure to the game, arousal responses differentiated between choices associated with positive and negative long-term outcomes, and occurred even before subjects were aware of such contingencies (Bechara, Damasio, Tranel, \& Damasio, 1997). Notably, a causal link between arousal and decisions was further corroborated by studies of patients with damage to the ventromedial prefrontal cortex, who were not able to integrate GSR responses into their decision-making process, and thus failed to learn the contingencies properly (Bechara, Damasio, Damasio, \& Lee, 1999; Bechara et al., 2001; Bechara, Tranel, \& Damasio, 2000). These studies are good examples of how measures of arousal states can inform us of physiological processes causally predictive of a subject's choices that the subject is not consciously aware of is taking place, and in some instances, may even run counter to the subject's self-awareness.

What is the reason for the ability of changes in physiological arousal to modulate preferences for objects, as well as choices? Damasio and Bechara's so-called "somatic marker" theory speculates that perception of biologically relevant information leads to changes to the interoceptive state of the body, changes that results in a number of physiological modifications, including changes to the arousal system (Bechara \& Damasio, 2005). Arousal, on its side, is known to impact the activity of the reward system that is thought to be the neural center of computing the values of objects, motivating preferences and decision behavior (Sokol-Hessner et al. 2009). For example, Pessiglione and colleagues recently reported that in gambles which subjects experienced as purely random, 
neural responses in the basal ganglia to subliminal conditioning predicted consumer risk taking (Pessiglione et al., 2008). Similarly, in the domain of consumer choice, studies by Knutson and his collaborators (e.g. Knutson, Rick, Wimmer, Prelec, \& Loewenstein, 2007) reports that responses in the ventral striatum during product viewing are positively related to subjects' subsequent willingness to buy (WTB), while responses of the anterior insula during price viewing are negatively related to WTB. Notably, while the temporal dimension allowed the researchers to claim that the neural responses predicted choice, this measure did not perform much better than merely asking the subjects about their expected choice. Here, a recent study by Berns \& Moore (2012) suggests that the activation in selected regions such as the ventral striatum in a small cohort may indeed predict the market response to products at a later time.

Physiological arousal is most often measured through pupil dilation measures or GSR. Besides found to be highly collinear measures (Bradley, Miccoli, Escrig, \& Lang, 2008), GSR and pupil dilation are well known to be measures of arousal and thus related to the engagement of brain structures such as the amygdala, ventromedial prefrontal cortex and insula, although this relationship is complex (Bechara et al., 1999; Pribram \& McGuinness, 1975; Raine, Reynolds, \& Sheard, 1991; Tranel \& Damasio, 1989). However, making such measures entail a problem. Changes in arousal measures do not distinguish between positive and negative emotional states. It is bivalent: arousal may shoot up both for strongly positive and strongly negative stimuli, and may thus serve as an index of the relevancy that an organism ascribes to a stimulus or an event (Bradley et al., 2008). Therefore, arousal measures need to be combined with other measures to provide a more complete picture of the emotional responses we have to events and stimuli. For example, anecdotal evidence suggests that we lean back when we see something aversive and negative, and we may lean forward when we see something positive and appealing. Indeed, in a recent study (Eerland, Guadalupe, Franken, \& Zwaan, 2012) it was shown that positive images were associated 
with leaning forward, and aversive images was related to leaning backwards. One possibility is that body posture may provide an index of approach and avoidance responses, and thus may provide an index of positive and negative valence, respectively.

Interestingly, high-resolution eye-tracking provides the opportunity of measuring pupil dilation, indexing arousal, and body posture at the same time. Therefore, eye-tracking experiments of preference formation or decision behavior could conceivably combine measures of body posture and pupil dilation to provide a more precise index of the valence of arousal. One way to avoid these effects is to include a pre-stimulus mask that has the same brightness as the stimulus image. This 'calibration' of pupil dilation is well-established in the literature and employed by leading eyetracking companies (e.g. iMotions Inc, see www.imotionsglobal.com) It should be noted, however, that the use of pupil dilation measures to assess arousal needs to pay specific heed to the nature of pupil dilation responses. First, pupils respond to brightness in a visual scene. Second, pupil dilation has long been known to be affected by task demands (Bradley, Miccoli, Escrig, \& Lang, 2008; Papesh \& Goldinger, 2011), but can be relatively easily adjusted for by using stimuli or tasks that are equally complex, or by assessing task complexity as a regressor in the analysis.

To address this assumption, we analyzed data from three different data sets using high-resolution eye-tracking during preference formation. In all cases, we collected measures of pupil dilation and physical distance from screen with a high temporal resolution. While study 1 was a study of the main effect of pupil dilation and body posture on consumer preference, study 2 serves as an alternative "defect" model take on this relationship, as this study measured the negative influence of simultaneously presented sounds on brand preference. In study 3 , we tested the predictive effect that the dynamic relationship between posture and pupil dilation could have on actual decision- 
making. Taken together, we assert that the results provide a more comprehensive understanding of the relationship between pupil dilation, body posture and value-based decision-making.

\section{Study 1}

This study focused on the effects of brands on preference for clothes. Besides the well known effect of brands on soft drinks (McClure et al., 2004), brands are known to affect preference for other consumer goods, including clothing, food items and cars (Plassmann et al., 2008; Reimann, Castaño, Zaichkowsky, \& Bechara, 2012; Santos, Seixas, Brandão, \& Moutinho, 2011; Schaefer \& Rotte, 2007; Schaefer, Berens, Heinze, \& Rotte, 2006; Schmitt, 2012; Shiv \& Yoon, 2012). Recent studies using neuroimaging methods have suggested that brands imbue products with value by recruiting memory-related processes (McClure et al., 2004) and reward related brain regions (Schaefer \& Rotte, 2007; Schaefer et al., 2006). Recently, studies have shown that brands affect emotional responses and subsequent emotional processing (see Plassmann, Ramsøy \& Milosavljevic, 2012, for an overview).

Both anecdotal and empirical evidence has demonstrated that preference judgments for branded objects are positively affected by individual brand preference (Bushman, 1993; Jamal \& Goode, 2001), and that such effects can even affect emotional processing of related information (Koeneke, Pedroni, Dieckmann, Bosch, \& Jäncke, 2008). As noted, measures of arousal, such as pupil dilation, have been related to value-based decision-making (Klebba, 1985; Preuschoff, Marius't Hart, \& Einhauser, 2011). Here, we set up a study to assess the effect of pupil dilation and body posture in predicting subsequent reports of product preference. Based on prior findings, we expected that pupil dilation and body posture would show individual main effects on preference judgments, and that effects of arousal would interact with the effects of body posture. 
Thirty women (age 25.1 \pm 2.9 years), all right handed and with normal or corrected to normal vision, were recruited from the Copenhagen region. They were instructed that they would be asked to rate their preference for clothing, as shown on the screen (see Figure 1). High-resolution eye-tracking was performed using a Tobii T60 XL tracker running at $60 \mathrm{~Hz}$ with a 1920x1200 pixel screen resolution and an approximate viewing distance of $60 \mathrm{~cm}$. Stimulus presentation, and the recording of subject responses and eye tracking data were performed using Attention Tool version 4.5 (iMotions Inc., www.imotionsglobal.com).

Figure 1

After undergoing a 9-point eye tracking calibration procedure, subjects saw a screen with a piece of fashion clothing, accompanied by a brand name, for 6 seconds. Prior to each image they saw a white-nosed version of the stimulus for 3 seconds. Each brand was a well-known high-fashion brand (e.g., Dior, Gucci, Prada) matched on subjective knowledge in a pilot study. The coupling between brands and outfits were randomized and counterbalanced for each subject, allowing for estimation of their individual effects. Following this screen subjects were asked to rate their preference for the clothing using an analogue scale on-screen, by using their right hand to control a computer mouse. The preference rating was self-paced. For each trial, we sampled both pupil dimension and distance to screen. Pupil dilation responses were sampled as the mean change in pupil diameter between the pre-stimulus period and the stimulus period. During the pre-stimulus period subjects saw a white noised image tailored to have the same brightness property as the stimulus image, a standard feature of the software used (Attention Tool v4.5). Distance was 
measured as a standard calculation of the Tobii T60XL tracker. This measure is performed through a triangulation of the known distance of each infrared eye-tracker sensor, and the distance between the eyes In total, each subject rated 32 different clothing products.

The statistical analysis was run in JMP version 9.0 (SAS Inc.). Preference judgment was used as the dependent variable, and we used pupil dilation, distance to screen, and dilation*distance interaction as independent factors. Subject and picture was used as random factors in the analysis, to take individual differences and picture luminosity effects into account

\section{Results}

The main model was significant $\left(\mathrm{R}^{2}=0.24, \mathrm{RMSE}=0.3822, \mathrm{p}<0.0001\right)$, and as Table 1 shows, pupil dilation and the interaction between pupil response and distance were significantly related to preference judgments. While pupil dilation was positively related to preference, the distance estimate showed no significant relationship to subsequent judgments.

Table 1

Figure 2

Our analysis also demonstrated a significant interaction between pupil dilation and distance to the screen. As Figure 2a shows, the relationship between pupil dilation and preference was affected by body posture: at low distance (i.e., subjects leaning slightly forward) there was a positive 
relationship between pupil dilation and preference; at high distance (i.e., leaning backwards), this relationship became negative, as stronger pupil dilation was related to lower preference ratings. Thus, pupil dilation responses and distance estimates make different and interactive contributions to predicting subsequent preference ratings.

However, the pupil dilation responses may be affected by changes in visual brightness caused by postural changes, which may cause systematic changes in pupil dilation responses. To test for this, we ran a post hoc exploratory regression analysis, using pupil dilation as the dependent variable and position as the independent variable. This analysis reveled a significant effect, although the explanatory value was low $\left(\mathrm{R}^{2}=0.029, \mathrm{RMSE}=291203.0, \mathrm{p}<0.0001\right)$. As posture only explained about $3 \%$ of the variation in pupil dilation we do not believe this effect violates the interpretation of our results.

\section{Discussion}

In this first study, we set out to test whether pupil dilation and body posture would produce different and interactive predictions of subsequent preference judgments. Here, we find that the two measures both produce individual predictions and interactive effects. On the one hand, pupil dilation showed a positive linear predictive effect on preference judgments. This corresponds to prior work relating increased arousal to positive alterations in consumer preference and choice (Groeppel-Klein, 2005). While, as we noted in the introduction, it has been demonstrated that arousal effects as such are orthogonal with respect to valence (Bradley et al., 2008; Dolcos, LaBar, \& Cabeza, 2004; Kousta, Vinson, \& Vigliocco, 2009), in the context of judgment of clothes, increased arousal is typically related to positive judgments, approach behavior and increased WTB.

Notably, our results clearly demonstrate that the correlation between pupil dilation and preference depends on body posture. When subjects were leaning forward, this relationship was positive, and 
stronger pupil dilation response was related to higher preference. However, when subjects were moving away from the screen, this relationship became negative, and stronger pupil dilation response was related to lower preference scores. Unfortunately, our results also show that pupil dilation was directly effected by postural distance, presumably due to the small changes in visual brightness. However, as posture only explained about 3\% of the variation in pupil dilation, we believe that we can rule out the possibility that postural changes have a strong impact on changes in visual brightness in the observer, and thus alterations in pupil dilation. Nevertheless, we suggest that further studies should be conducted to provide further specific tests of this, as well as means to correct for this as a potential artifact.

Study 1 provides a clear demonstration that pupil dilation and body posture can provide conjoined added value in predicting and understanding preference judgments. While posture did not provide any significant predictive value alone, posture had a modulatory role in the effect of pupil dilation. To our knowledge, this is the first study to demonstrate a combined value of arousal (such as pupil dilation) and valence (tentatively body posture) in understanding and predicting judgment and preferences.

It should be noted that further studies should include other data, such as fixation time and gaze, to test for added explanatory effects of posture to traditional eye-tracking measures. In this study, our focus on pupil dilation responses led us to explicitly design the studies to provide relatively stable fixations to a single brand label at a time, and not in competition with other stimuli. This excludes fixation as a parameter in the current experimental designs. Moreover, it should be noted that fixation data are highly related/collinear with emotional arousal. After all, emotions are well known to engage attention (Serences, 2008). 
As said, increased arousal may also be due to aversive events, such as disgusting images, punishment and other adverse events (Kousta et al., 2009; Nasrallah, Carmel, \& Lavie, 2009). Thus, if the context of judgment entails a more negative situation, it is likely that the independent and conjoint roles of pupil dilation and posture may be altered. To probe the predictive value of arousal and distance in a context of negative stimuli, we analyzed the dynamic relationship between posture and pupil dilation effects from a recently published stud (Ramsøy, Friis-Olivarius, Jacobsen, Jensen, \& Skov, 2012) where we used unpredictable sounds to induce a negative arousal effect. In prior studies, this has been demonstrated to work as a contextual uncertainty that induces changes in amygdala activation and avoidance behavior (Herry et al., 2007; Ramsøy et al. 2012).

\section{Study 2}

Eighteen right-handed subjects (age $24.8 \pm 1.5$ years, 8 women) with normal or corrected to normal vision were recruited from the Copenhagen region. Based on a self-report questionnaire, no subject suffered from, or had any indication of, neurological or psychiatric disease. All subjects provided informed consent. As in Study 1, we employed high-resolution eye-tracking with the same specifications and assessment parameters.

Figure 3

During the task, subjects saw a novel brand logo for three seconds. One second before the logo image was displayed subjects heard a simple sound sequence, which also lasted throughout the 3second logo image presentation. The sound was either played in a predictable or an unpredictable sequence, according to recent studies (Herry et al., 2007, Ramsøy et al., 2012). As in Study 1, we 
sampled both pupil dimension and distance to the screen. After the presentation of a brand logo, subjects were asked to rate their preference for the item on an analogue scale on the screen using a computer mouse (see Figure 3).

Novel brand logos were chosen due to expected stronger arousal effects compared to well-known brand logos, that may lead to more habituated responses that were less affected by non-visual stimuli. As in study 1 all brand logos had been pretested in a pilot study using a different cohort, and to further ensure brand logo novelty, we included a debriefing questionnaire on prior knowledge of the brand logo. No brand logos were previously known by the subjects.

All data were analyzed using JMP version 9.0 (SAS lnc.). We used the preference score as the dependent variable, and with pupil dimension, distance to screen and their interaction as independent factors. Subject was included as a random factor, and sound type was used as a covariate of no interest. Notably, as we assumed different effects on arousal during pure tone stimulation and the tone plus image condition, we ran the analysis separately for each condition.

\section{Results}

Our first analysis focused on the combined effects of pupil dilation and distance from the screen in predicting subsequent preference ratings, for the pure sound condition and the sound plus image condition separately. For the pure sound condition, the overall model was significant $\left(\mathrm{R}^{2}=0.20\right.$, RMSE=0.3856, $\mathrm{p}<0.0001$ ). As shown in Table 2, our general linear model (GLM) analysis demonstrated a significant negative effect of pupil dilation. That is, larger pupil size was related to lower subsequent preference ratings. While posture did not show any significant effect on preference, there was a significant interaction between posture and pupil dilation. As Figure 4a shows, pupil dilation at high distance was negatively related to preferences, while pupil dilation at low distance showed no relationship to preference. In other words, during pure sound perception, 
leaning forward has no additional positive effect on the relationship between pupil dilation and preference.

\section{TABLE 2}

Looking at the effect of pupil dilation and posture on preference when subjects both heard the sound and saw the logo image, we find that all parameters are significant and a significant overall model $\left(\mathrm{R}^{2}=0.19, \mathrm{RMSE}=0.3932, \mathrm{p}<0.0001\right)$. As Table 2 shows, larger pupil size was significantly related to higher preference scores; higher screen distance was related to lower preference scores, and we find a significant interaction between pupil size and distance to the screen. As shown in Figure $4 b$, the relationship between pupil dilation and preference was positive when subjects were leaning forward, and negative when they were leaning backwards.

Figure $4 \mathrm{a}+\mathrm{b}$

\section{Discussion}

The aim of Study 2 was to test whether the predictive effects of pupil dilation and body posture on preference judgments would be affected by negative contextual effects. As with Study 1, our data demonstrate a significant positive relationship between pupil dilation and preference judgments, and a negative trend of distance to the screen and judgments. However, the data from Study 2 
provide novel insights into the added value of pupil dilation and body posture. In particular, the arousal and posture effects were different for the two phases of the experiment. When subjects both saw the image they were expected to judge and heard the sound, we found the same modulation of body posture on the relationship between pupil dilation and preference (Figure 4b). That is, at low distance, pupil size was positively related to preference. At high distance, this relationship became negative. This lends further support to our findings in Study 1, which demonstrated the very same effect.

Conversely, in the first phase, when only the sound was presented, we found a change in the effects. Here, low distance (i.e., leaning forward) was not associated with a positive relationship between pupil dilation and preference. At high distance, we find the same negative relationship between pupil dilation and preference. This suggests that the effects induced by unpredictable sounds were associated with the reduction or elimination of the positive relationship between pupil dilation and preference. Nevertheless, as the present experimental design was not intended to test the independent effects of sound only and sound plus brand, these results must be treated with caution.

Studies 1 and 2 demonstrate that a model using the interaction between pupil dilation and posture can have additional predictive power of subsequent preference judgments. However, this still leaves open the question of whether the same method predicts actual choice behaviors. Therefore, a third study was conducted to test the predictive ability of combined posture and pupil data on consumer choice.

\section{Study 3}

In this study, 98 subjects (age 22.6 $\pm 1.5,52$ males) were recruited from the Copenhagen region. Similar enrollment procedures were conducted as in the two previous studies, and no subjects were 
rejected based on self-report neurological or psychiatric disorder. All subjects provided informed consent. The study conducted employed high-resolution eye-tracking using a Tobii T60 XL tracker running with Attention Tool.

\section{FIGURE 5}

This experiment focused on subjects' willingness to pay (WTP) for branded wine. Prior to the experiment, subjects were endowed with 200 Danish Kroner (DKK, approximately US\$36) to be used in the experiment, or saved for cash payment. Subjects first saw an image of a wine brand and the country of origin for 6 seconds. Subjects were then instructed to select and taste wine from a small numbered cup with $10 \mathrm{cl}$ of wine. They then pressed a button when they had swallowed the wine, and proceeded to rate their experience of the wine taste by using an on-screen visual analogue scale. Between each wine tasting round, participants rinsed their mouth with water. After the trial, subjects were presented with the brand logos again, and were asked to report their WTP for that particular wine. Subjects were instructed that their WTP choices would be effectuated through a lottery in which their choices would be randomly selected, and the wine receiving the highest bid would be realized. Should the highest bid not amount to 200 DKK, they would be paid the remaining amount through bank transfer. This meant that subjects were motivated to optimize their wine choices, which allowed a better estimation of actual WTP, rather than subjective estimates of WTP. 
In all, six branded red wines were presented twice in a fixed pseudorandom order, but unbeknownst to the subject, they were only served the same wine, which was neither of the branded wines. During each tasting, we recorded pupil dilation responses and postural changes.

In a random effects regression analysis, we tested the effect of pupil dilation, posture and their interaction on predicting WTP during the Branding phase, i.e. when only brand related information was presented. Subject was used as random factor. Our experimental setup allowed a direct comparison of the distinct explanatory values of self-reported liking and physiological measures. To obtain parametric distribution of WTP data, they were $\log$ transformed $(\log$ WTP), and all subsequent analyses are made on this value. This was done by first making a full random effects analysis with $\log$ WTP as the dependent variable, and first including all factors (liking, pupil dilation, posture, and pupil*posture interaction) as independent variables. We then tested the explanatory power of liking and physiology separately. In all cases, we used $\mathrm{R}^{2}$ as a standardized estimate of the explanatory power of the random effects model.

\section{Results}

Subjects showed a willingness to pay of 84.7 DKK on average per wine, but there was also a large range between wines ( $\mathrm{SD}=63.4 \mathrm{DKK}$, range 0-300 DKK). Also, individuals differed on average WTP scores $(\mathrm{df}=97, \mathrm{~F}=1979.9, \mathrm{p}<0.0001)$.

In the Branding phase, we find that pupil dilation, posture and the pupil*posture interaction are all significant predictors of subsequent WTP (see Table 3) 
TABLE 3

\begin{tabular}{|l|r|r|r|r|}
\hline Source & DF & DFDen & $\mathbf{F}$ & $\mathbf{p}$ \\
\hline Pupil dilation & 1 & $2.00 \mathrm{E}+05$ & 1254.3 & $<.0001$ \\
\hline Posture & 1 & $1.00 \mathrm{E}+05$ & 61.8 & $<.0001$ \\
\hline Pupil * Posture & 1 & $2.00 \mathrm{E}+05$ & 322.0 & $<.0001$ \\
\hline
\end{tabular}

Our second approach was to test and compare the explanatory effects that self-reported liking and physiology measures would have on WTP. Here, we find that the full model with all factors had an explanatory value of $31 \%\left(\mathrm{R}^{2}=0.310, \mathrm{RMSE}=0.605, \mathrm{p}<0.0001\right)$. The explanatory power of liking alone was $29.5 \%\left(\mathrm{R}^{2}=0.295, \mathrm{RMSE}=0.599, \mathrm{p}<0.0001\right)$, while for physiology (pupil dilation, posture, and pupil*posture interaction $)$ the explanatory power was $27.6 \%\left(\mathrm{R}^{2}=0.276, \mathrm{RMSE}=0.611\right.$, $\mathrm{p}<0.0001)$

Finally, an exploratory analysis was run to test the relationship between physiological measures and subjective liking reports. Using a random effects analysis with liking as the dependent variable and pupil dilation, posture, and pupil*posture interaction as independent variables. This analysis conveyed that although the effect of pupil dilation response $(F=478.7, p<0.0001)$, posture $(F=34.4$, $\mathrm{p}<0.0001)$ and their interaction $(\mathrm{F}=80.3, \mathrm{p}<0.0001)$ had a significant relationship with liking, the overall explanatory value of the combined physiological measures was relatively low $\left(\mathrm{R}^{2}=0.063\right.$, $\mathrm{RMSE}=158.9, \mathrm{p}<0.0001)$.

\section{Discussion}

The goal of this third study was to test whether the independent and joint contributions of pupil dilation responses and posture could explain subsequent choice. By allowing subjects to bid for 
products after a trial had ended, we find that pupil dilation, posture and their interaction were significantly predictive of subsequent choice, as assessed by WTP scores. By comparing the explanatory power of these physiology measures and self-reported liking during the trial, we found similar explanatory powers of both measures, and only incremental added value of a full model which includes both self-report and physiology measures.

Two main conclusions can be drawn on the basis of this study. First, measures of physiological responses and self-reported preference seem to have comparable predictive powers with respect to choice. This suggests that the two measures can be used interchangeably. This finding is highly reminiscent of previous studies, especially a study by Knutson et al. (Knutson, Rick, Wimmer, Prelec, \& Loewenstein, 2007) in which neural responses in the brain's ventral striatum during product viewing was predictive of purchase behavior 8-12 seconds later. However, when compared to self-reported preference reports, neural responses had little additional predictive power on subsequent choice. Nevertheless, these findings imply that neural responses can be significantly predictive of actual choice occurring several seconds later. Others have demonstrated that such early physiological responses are more predictive of choice than self reports. For example, Berns \& Moore (2012) report that the neural specific activation in a group of teenagers listening to music predicted the cultural popularity of the music two years later. Self-reported liking had a much lower predictive value on this effect. The current finding positions it in this framework by suggesting that the assessment of pupil dilation and posture during brand (or product) display may predict subsequent choice behaviors, and that this effect could be found when only the brands had been perceived, and thus before any product was presented.

Such findings - neuroimaging and neurophysiology alike - may be useful for several purposes. Most notably, there may be many instances in which overt liking reports are unavailable or not 
possible to collect. Furthermore, if physiological responses are predictive of choice behaviors, they may be used as an early means to sample and possibly intervene on choice behaviors. Such interventions could be of interest both from commercial and clinical approaches.

Second, it should be noted that our data does not suggest that there is any particular added value of using both methods in predicting subsequent WTP. One possible interpretation of this is a causal model in which direct physiological responses to brands has a strong effect on subsequent subjective rating. However, as our post-hoc analysis showed, the actual relationship between physiology and liking was relatively low, implying that physiology and overt liking make different contributions to subsequent willingness to pay.

\section{General discussion}

The aim of this study was to test two questions: one scientific and one technical. First, we wanted to test whether the assessment of body posture would increase the predictive value of arousal measures such as pupil dilation with respect to judgment and decision-making. Second, we were interested in testing whether the data provided from recent high-resolution eye-tracking could reliably be used to this end. In three unrelated studies we demonstrate that the predictive value of pupil dilation on preference judgments and choice is modulated by measures of body posture. Specifically, we find that during viewing of fashion clothing, pupil dilation in general was positively related to preference. This relationship was significantly modulated by body posture: when subjects were leaning forward, pupil dilation showed a strong positive relationship with preference. However, when subjects were leaning backwards, pupil dilation showed an inverse relationship, i.e., stronger pupil dilation response was negatively related to preference. This suggests 
that pupil dilation and posture can be used in combination to assess and predict consumer preference.

While our second study confirmed this relationship during visual perception, we also found that the effect of aversive tones during pure sound presentation altered this relationship. In particular, we found the same negative relationship between pupil dilation and preference when subjects were leaning backwards. However, the results demonstrated that when subjects were leaning forward, the positive relationship between pupil dilation and preference was abolished. These findings suggests that the sound abolished the positive relationship between pupil dilation and preference, and tentatively enhanced the adverse effect pupil size had on experience and judgment.

In our final study, we link the interaction between posture and arousal to actual decision-making. Here, our data show that pupil dilation and posture have both independent and interactive contributions in the prediction of subjects' willingness to pay for the products. In particular, we find that the actual relationship between these physiological measures and subjective preference ratings, although providing comparable contributions to WTP, were not collinear. This suggests that, perhaps not surprisingly, physiological measures early in the consumer decision process differ from subjective ratings after the branding and tasting has taken place.

These findings provide novel insights into the bodily responses accompanying consumer perception, emotional response, judgment and choice. Taken together, the results imply that we should use body posture as an additional index to assess the relative valence that is associated with particular pupil dilation responses. In this vein, our data provide a clear demonstration that assessment with high-resolution eye tracking can be used to assess both pupil dilation responses and posture in understanding consumer preference and choice. 
To our knowledge, this is the first study to combine pupil dilation and posture in understanding consumer choice, and in understanding preference in general. Consequently, more research is needed for several reasons, of which we here name only a few: (1) Is the pupil-posture interaction valid across different conditions, products and choice types? Can we, for example, employ this method in testing advertisement responses, financial risk taking and social choices? (2) Can the pupil-posture index be used to understand aberrant consumer behaviors? For example, can it be used to better understand emotional responses in compulsive buying behavior and pathological gambling? And, finally: (3) What are the underlying neural mechanisms of the pupil-posture interaction? Is it possible that approach-related posture is related to one kind of neural activation (e.g., the medial orbitofrontal cortex) as opposed to avoidance-related posture (e.g., anterior insula)? How do these and other, more common neural processes, influence the effect that pupil dilation has on preference and choice?

As a final note, we should mention that further studies need to test the added value of other data that are available in eye-tracking data. First, it is known that data on fixation time and gaze may be predictive of preference and choice (Wedel \& Pieters, 2000). While this is an interesting point that deserves a separate study, we chose not to include fixation data for several reasons. Most importantly, our focus on pupil dilation responses led us to explicitly design the studies to provide relatively stable fixations to a single brand label at a time, and not in competition with other stimuli. This excludes fixation as a parameter in the current experimental designs. Moreover, fixation data are highly related/collinear with emotional arousal. After all, emotions are well known to engage attention, and thus we believe that there would be little added value of adding fixation time and gaze for our present study (Lang \& Bradley, 2010; Olofsson, Nordin, Sequeira, \& Polich, 2008; Pessoa, 2009). 
Thus, we believe that the present study, by holding some parameters constant, allow us to make conclusions about whether posture data can improve our predictive model. In particular, we contend that the usual limitations of pupil dilation associated with bivalent arousal can be informed by including posture data. It seems that when leaning forward, pupil dilation is related to stronger preference and increased likelihood of purchase. Conversely, when leaning backwards increased pupil dilation is negatively related to preference and purchase. 


\section{References}

Ariely, D., \& Berns, G. S. (2010). Neuromarketing: The hope and hype of neuroimaging in business. Nature Reviews. Neuroscience, 11(4), 284-92. doi:10.1038/nrn2795

Bechara, A., Damasio, H., Damasio, A. R., \& Lee, G. P. (1999). Different contributions of the human amygdala and ventromedial prefrontal cortex to decision-making. The Journal of Neuroscience : The Official Journal of the Society for Neuroscience, 19(13), 5473-81.

Bechara, A., Damasio, H., Tranel, D., \& Damasio, A. R. (1997). Deciding advantageously before knowing the advantageous strategy. Science, 275(5304), 1293-5.

Bechara, A., Dolan, S., Denburg, N., Hindes, A., Anderson, S. W., \& Nathan, P. E. (2001). Decision-making deficits, linked to a dysfunctional ventromedial prefrontal cortex, revealed in alcohol and stimulant abusers. Neuropsychologia, 39(4), 376-89.

Bechara, A., Tranel, D., \& Damasio, H. (2000). Characterization of the decision-making deficit of patients with ventromedial prefrontal cortex lesions. Brain : A Journal of Neurology, 123 ( Pt 11), 2189-202.

Berns, G. S., \& Moore, S. E. (2012). A neural predictor of cultural popularity. Journal of Consumer Psychology, 22(1), 154-160. doi:10.1016/j.jcps.2011.05.001 
Bradley, M. M., Miccoli, L., Escrig, M. A., \& Lang, P. J. (2008). The pupil as a measure of emotional arousal and autonomic activation. Psychophysiology, 45(4), 602-7. doi:10.1111/j. 1469-8986.2008.00654.x

Braeutigam, S. (2005). Neuroeconomics--from neural systems to economic behaviour. Brain Research Bulletin, 67(5), 355-60. doi:10.1016/j.brainresbull.2005.06.009

Bushman, B. J. (1993). What's in a name? The moderating role of public self-consciousness on the relation between brand label and brand preference. Journal of Applied Psychology, 78(5), 857.

Camerer, C., Loewenstein, G., \& Prelec, D. (2005). Neuroeconomics: How neuroscience can inform economics. Journal of Economic Literature, 43(1), 9-64.

Churchland, P. S. (1981). The timing of sensations: Reply to libet. Philosophy of Science, 48(3), 492-497.

Dolcos, F., LaBar, K. S., \& Cabeza, R. (2004). Dissociable effects of arousal and valence on prefrontal activity indexing emotional evaluation and subsequent memory: An event-related fmri study. NeuroImage, 23(1), 64-74. doi:10.1016/j.neuroimage.2004.05.015

Eerland, A., Guadalupe, T. M., Franken, I. H., \& Zwaan, R. A. (2012). Posture as index for approach-avoidance behavior. PloS One, 7(2), e31291. doi:10.1371/journal.pone.0031291 
Fisher, C. E., Chin, L., \& Klitzman, R. (2010). Defining neuromarketing: Practices and professional challenges. Harvard Review of Psychiatry, 18(4), 230-7. doi:10.3109/10673229.2010.496623

Groeppel-Klein, A. (2005). Arousal and consumer in-store behavior. Brain Research Bulletin, 67(5), 428-37. doi:10.1016/j.brainresbull.2005.06.012

Herry, C., Bach, D. R., Esposito, F., Di Salle, F., Perrig, W. J., Scheffler, K., . . Seifritz, E. (2007). Processing of temporal unpredictability in human and animal amygdala. The Journal of Neuroscience : The Official Journal of the Society for Neuroscience, 27(22), 5958-66. doi:10.1523/ JNEUROSCI.5218-06.2007

Hubert, M., \& Kenning, P. (2008). A current overview of consumer neuroscience. Journal of Consumer Behaviour, 7(4-5), 272-292. doi:10.1002/cb.251

Jamal, A., \& Goode, M. M. H. (2001). Consumers and brands: A study of the impact of self-image congruence on brand preference and satisfaction. Marketing Intelligence \& Planning, 19(7), 482-492.

Kenning, P., \& Plassmann, H. (2005). Neuroeconomics: An overview from an economic perspective. Brain Research Bulletin, 67(5), 343-54. doi:10.1016/j.brainresbull.2005.07.006

Klebba, J. M. (1985). Physiological measures of research: A review of brain activity, electrodermal response, pupil dilation, and voice analysis methods and studies. Current Issues and Research in Advertising, 8(1), 53-76. 
Klein, S. A. (2002). Libet's temporal anomalies: A reassessment of the data. Consciousness and Cognition, 11(2), 198-214. doi:10.1006/ccog.2002.0573

Knutson, B., Rick, S., Wimmer, G. E., Prelec, D., \& Loewenstein, G. (2007). Neural predictors of purchases. Neuron, 53(1), 147-56. doi:10.1016/j.neuron.2006.11.010

Koeneke, S., Pedroni, A. F., Dieckmann, A., Bosch, V., \& Jäncke, L. (2008). Individual preferences modulate incentive values: Evidence from functional MRI. Behavioral and Brain Functions : BBF, 4, 55. doi:10.1186/1744-9081-4-55

Kousta, S. T., Vinson, D. P., \& Vigliocco, G. (2009). Emotion words, regardless of polarity, have a processing advantage over neutral words. Cognition, 112(3), 473-81. doi:10.1016/j.cognition. 2009.06.007

Lang, P. J., \& Bradley, M. M. (2010). Emotion and the motivational brain. Biological Psychology, 84(3), 437-50. doi:10.1016/j.biopsycho.2009.10.007

Lee, N., Broderick, A. J., \& Chamberlain, L. (2007). What is "neuromarketing"? A discussion and agenda for future research. International Journal of Psychophysiology : Official Journal of the International Organization of Psychophysiology, 63(2), 199-204. doi:10.1016/j.ijpsycho. 2006.03.007

Levita, L., Hare, T. A., Voss, H. U., Glover, G., Ballon, D. J., \& Casey, B. J. (2009). The bivalent side of the nucleus accumbens. NeuroImage, 44(3), 1178-87. doi:10.1016/j.neuroimage. 2008.09.039 
Libet, B. (1999). How does conscious experience arise? The neural time factor. Brain Research Bulletin, 50(5-6), 339-40.

Libet, B. (2002). The timing of mental events: Libet's experimental findings and their implications. Consciousness and Cognition, 11(2), 291-9; discussion 304-33.

Libet, B., Gleason, C. A., Wright, E. W., \& Pearl, D. K. (1983). Time of conscious intention to act in relation to onset of cerebral activity (readiness-potential). Brain : A Journal of Neurology, $106(3), 623-642$.

McClure, S. M., Li, J., Tomlin, D., Cypert, K. S., Montague, L. M., \& Montague, P. R. (2004). Neural correlates of behavioral preference for culturally familiar drinks. Neuron, 44(2), 379-87. doi: 10.1016/j.neuron.2004.09.019

Mohr, P. N., Biele, G., Krugel, L. K., Li, S. C., \& Heekeren, H. R. (2010). Neural foundations of risk-return trade-off in investment decisions. NeuroImage, 49(3), 2556-63. doi:10.1016/ j.neuroimage.2009.10.060

Murray, E. A. (2007). The amygdala, reward and emotion. Trends in Cognitive Sciences, 11(11), 489-97. doi:10.1016/j.tics.2007.08.013

Nasrallah, M., Carmel, D., \& Lavie, N. (2009). Murder, she wrote: Enhanced sensitivity to negative word valence. Emotion (Washington, D.C.), 9(5), 609-18. doi:10.1037/a0016305 
Olofsson, J. K., Nordin, S., Sequeira, H., \& Polich, J. (2008). Affective picture processing: An integrative review of ERP findings. Biological Psychology, 77(3), 247-65. doi:10.1016/j.biopsycho. 2007.11.006

Papesh, M. H., \& Goldinger, S. D. (2011). Your effort is showing! Pupil dilation reveals memory heuristics. Constructions of Remembering and Metacognition. Palgrave Macmillan, 215-224.

Pessiglione, M., Petrovic, P., Daunizeau, J., Palminteri, S., Dolan, R. J., \& Frith, C. D. (2008). Subliminal instrumental conditioning demonstrated in the human brain. Neuron, 59(4), 561-7. doi: 10.1016/j.neuron.2008.07.005

Pessoa, L. (2009). How do emotion and motivation direct executive control? Trends in Cognitive Sciences, 13(4), 160-6. doi:10.1016/j.tics.2009.01.006

Plassmann, H., Kenning, P., Deppe, M., Kugel, H., \& Schwindt, W. (2008). How choice ambiguity modulates activity in brain areas representing brand preference: Evidence from consumer neuroscience. Journal of Consumer Behaviour, 7(4-5), 360-367. doi:10.1002/cb.257

Plassmann, H., Ramsøy, T. Z., \& Milosavljevic, M. (2012). Branding the brain: A critical review and outlook. Journal of Consumer Psychology, 22(1), 18-36. doi:10.1016/j.jcps.2011.11.010

Preuschoff, K., Marius’t Hart, B., \& Einhauser, W. (2011). Frontiers: Pupil dilation signals surprise: Evidence for noradrenaline's role in decision making. Frontiers in Decision Neuroscience, 5. 
Pribram, K. H., \& McGuinness, D. (1975). Arousal, activation, and effort in the control of attention. Psychological Review, 82(2), 116-49.

Raine, A., Reynolds, G. P., \& Sheard, C. (1991). Neuroanatomical correlates of skin conductance orienting in normal humans: A magnetic resonance imaging study. Psychophysiology, 28(5), 548-58.

Ramsøy, T. Z., Friis-Olivarius, M., Jacobsen, C., Jensen, S. B., \& Skov, M. (2012). Effects of perceptual uncertainty on arousal and preference across different visual domains. Journal of Neuroscience, Psychology, and Economics, 5(4), 212-226. doi:10.1037/a0030198

Reimann, M., Castaño, R., Zaichkowsky, J., \& Bechara, A. (2012). How we relate to brands: Psychological and neurophysiological insights into consumer-brand relationships. Journal of Consumer Psychology, 22(1), 128-142. doi:10.1016/j.jcps.2011.11.003

Rustichini, A. (2005). Neuroeconomics: Present and future. Games and Economic Behavior, 52(2), 201-212. doi:10.1016/j.geb.2005.05.004

Santos, J. P., Seixas, D., Brandão, S., \& Moutinho, L. (2011). Investigating the role of the ventromedial prefrontal cortex in the assessment of brands. Frontiers in Neuroscience, 5, 77. doi: 10.3389/fnins.2011.00077

Schaefer, M., \& Rotte, M. (2007). Thinking on luxury or pragmatic brand products: Brain responses to different categories of culturally based brands. Brain Research, 1165, 98-104. doi:10.1016/ j.brainres. 2007.06 .038 
Schaefer, M., Berens, H., Heinze, H. J., \& Rotte, M. (2006). Neural correlates of culturally familiar brands of car manufacturers. NeuroImage, 31(2), 861-5. doi:10.1016/j.neuroimage.2005.12.047

Schmitt, B. (2012). The consumer psychology of brands. Journal of Consumer Psychology, 22(1), 7-17. doi:10.1016/j.jcps.2011.09.005

Senior, C., \& Lee, N. (2008). A manifesto for neuromarketing science. Journal of Consumer Behaviour, 7(4-5), 263-271. doi:10.1002/cb.250

Serences, J. T. (2008). Value-based modulations in human visual cortex. Neuron, 60(6), 1169-81. doi:10.1016/j.neuron.2008.10.051

Shiv, B., \& Yoon, C. (2012). Integrating neurophysiological and psychological approaches: Towards an advancement of brand insights. Journal of Consumer Psychology, 22(1), 3-6. doi:10.1016/j.jcps. 2012.01.003

Sokol-Hessner, P., Hsu, M., Curley, N. G., Delgado, M. R., Camerer, C. F., \& Phelps, E. A. (2009). Thinking like a trader selectively reduces individuals' loss aversion. Proceedings of the National Academy of Sciences of the United States of America, 106(13), 5035-40. doi:10.1073/pnas. 0806761106

Soon, C. S., Brass, M., Heinze, H. J., \& Haynes, J. D. (2008). Unconscious determinants of free decisions in the human brain. Nature Neuroscience, 11(5), 543-5. doi:10.1038/nn.2112 
Tranel, D., \& Damasio, H. (1989). Intact electrodermal skin conductance responses after bilateral amygdala damage. Neuropsychologia, 27(4), 381-90.

Wedel, M., \& Pieters, R. (2000). Eye fixations on advertisements and memory for brands: A model and findings. Marketing Science, 297-312. Retrieved from Google Scholar.

Wunderlich, K., Rangel, A., \& O'Doherty, J. P. (2010). Economic choices can be made using only stimulus values. Proceedings of the National Academy of Sciences of the United States of America, 107(34), 15005-10. doi:10.1073/pnas.1002258107 


\section{FIGURE CAPTIONS}

Figure 1

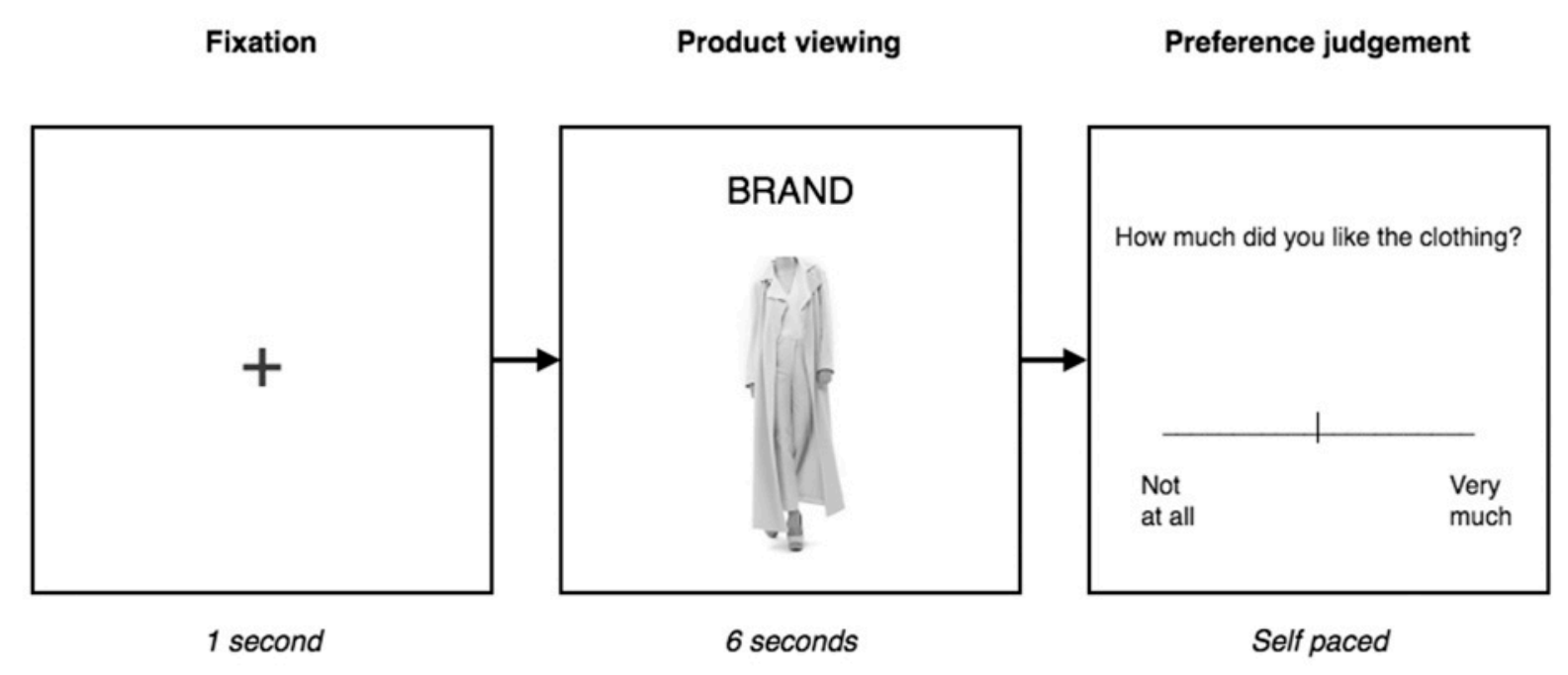

Study design for Study 1. Subjects first saw a fixation cross, followed by a display with a piece of clothing, where the brand name was displayed on top of the clothing. After each image, subjects were asked to report their preference for the clothing using an analogue scale by controlling a computer mouse. 
Figure 2
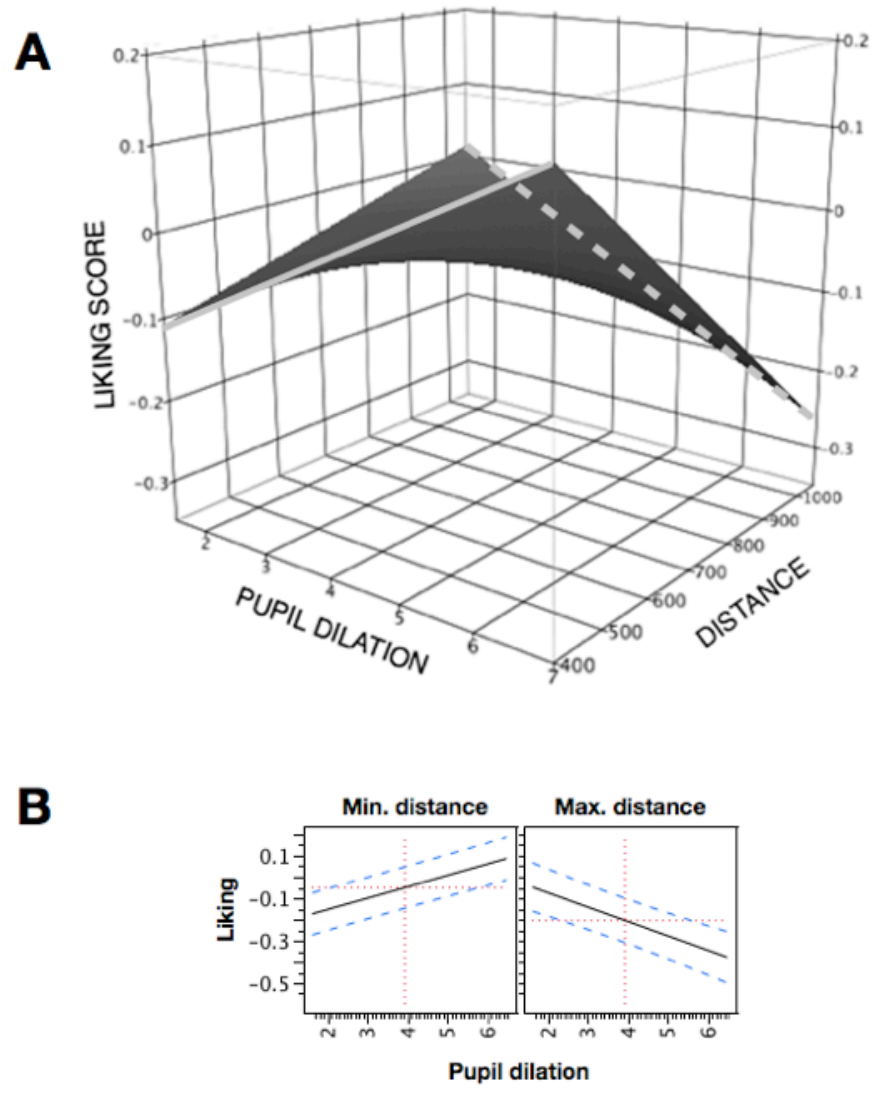

Relationship between pupil size and posture in predicting subsequent product judgment, using a 3D model (A) and illustrating using the extreme versions of leaning forward and backwards, where minimum distance $=51.2 \mathrm{~cm}$, and maximum distance $=80.5 \mathrm{~cm}(\mathrm{~B})$. As evident in the figure, there was a positive relationship between pupil dilation and preference when subjects were leaning forward (i.e., low distance scores). This relationship became inverse and negative when subjects were leaning backward (i.e., high distance scores). Thus, high liking was predicted by increasing pupil dilation and low distance to the screen was at a minimum. Conversely, high pupil dilation and high distance was related to the lowest liking score. Notably, high liking score was also obtained for low pupil dilation and high distance. 


\section{Figure 3}

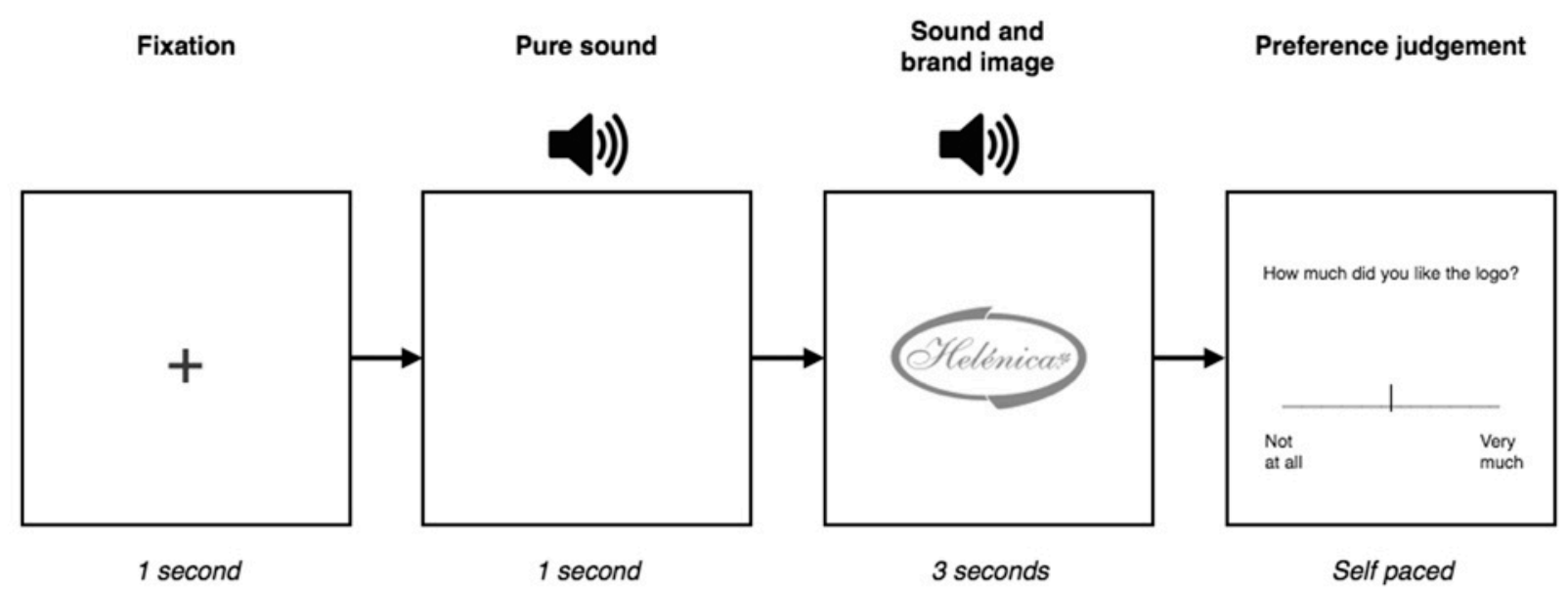

Experimental set-up for Study 2. Subjects first saw a fixation cross, for a first 1 second, a sound sequence (predictable or unpredictable) was played, after which a brand logo image was displayed for 3 seconds while the sound continued. Subjects were then asked to rate their preference for the brand logo using a visual analogue scale. 
Figure 4

A

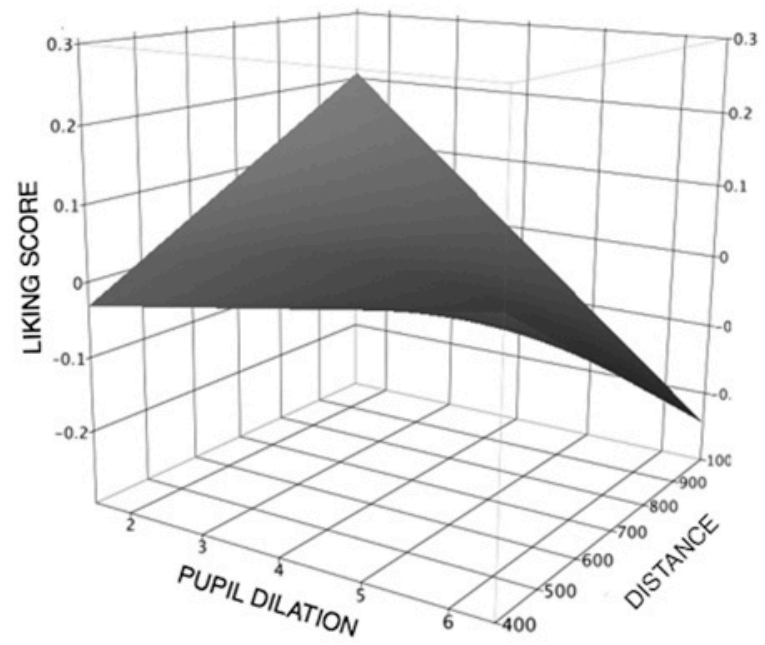

B

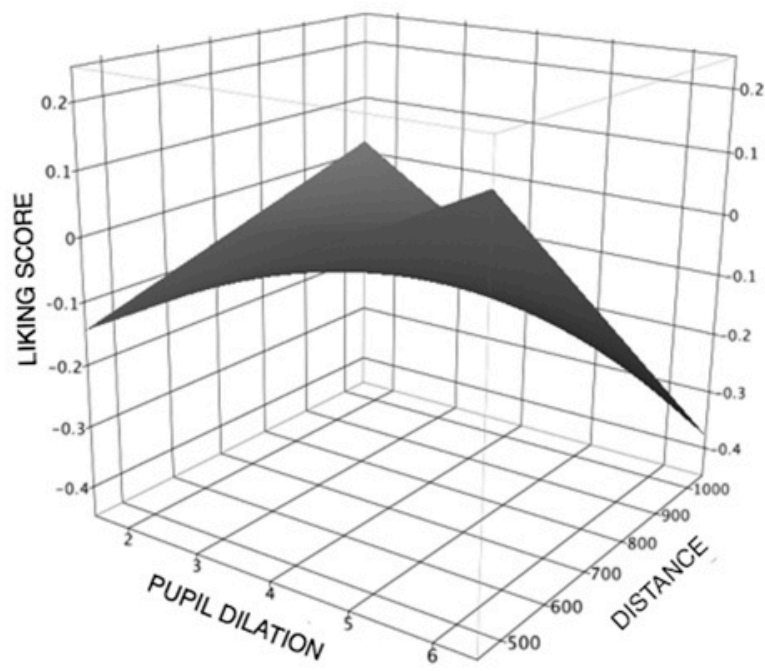

Three-way effects of pupil dilation and posture on subsequent preference judgment, at two different stimulus phases in Study 2. During pure sound perception (A) the effect of pupil size on judgment was only present at high distance, displaying a negative relationship between posture and preference. During the combined logo image and sound phase (B) changes in body posture were associated with changes in the relationship between pupil size and preference. At lower distance, pupil size was positively related to preference, while when subjects were leaning backwards, pupil size was negatively related to preference. 
Figure 5

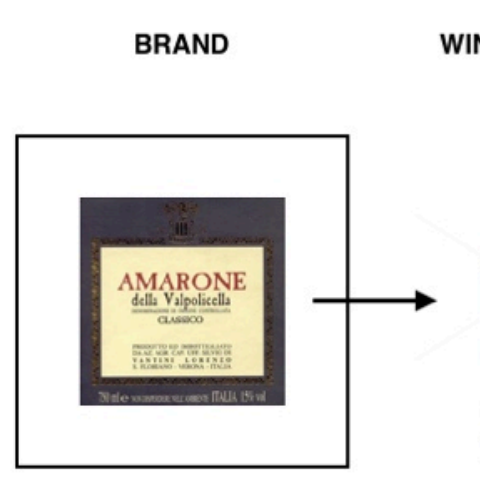

4 second
WINE TASTING

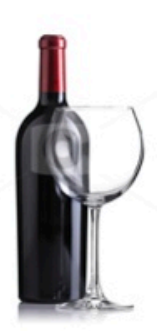

Self paced

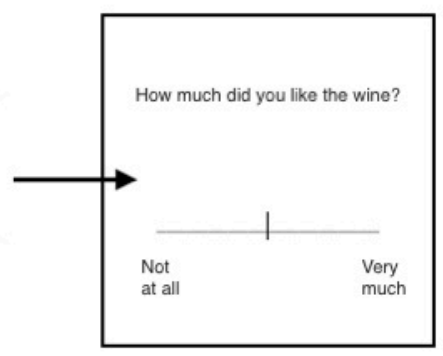

Self paced
WILLINGNESS TO PAY

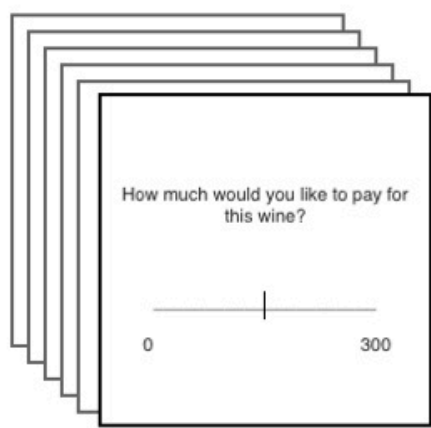

Self paced

Experimental setup for Study 3. Subjects first saw a wine brand, after which they were asked to taste the wine and make a rating of their liking of the product. After the end of all trials, subjects were shown the brand, and asked how much they would be willing to pay for taking that particular brand of wine with them after the experiment was over. 